\title{
LETTER \\ Dynamically Constrained Vector Field Convolution for Active Contour Model
}

\author{
Guoqi LIU ${ }^{\dagger \mathrm{a})}$, Zhiheng ZHOU ${ }^{\dagger \mathrm{b})}$, Shengli $\mathrm{XIE}^{\dagger \mathrm{c})}$, Nonmembers, and Dongcheng WU ${ }^{\dagger * \mathrm{~d})}$, Member
}

\begin{abstract}
SUMMARY Vector field convolution (VFC) provides a successful external force for an active contour model. However, it fails to extract the complex geometries, especially the deep concavity when the initial contour is set outside the object or the concave region. In this letter, dynamically constrained vector field convolution (DCVFC) external force is proposed to solve this problem. In DCVFC, the indicator function with respect to the evolving contour is introduced to restrain the correlation of external forces generated by different edges, and the forces dynamically generated by complex concave edges gradually make the contour move to the object. On the other hand, traditional vector field, a component of the proposed DCVFC, makes the evolving contour stop at the object boundary. The connections between VFC and DCVFC are also analyzed. DCVFC maintains desirable properties of VFC, such as robustness to initialization. Experimental results demonstrate that DCVFC snake provides a much better segmentation than VFC snake.

key words: active contour model, vector field convolution, correlation, snakes, image segmentation
\end{abstract}

\section{Introduction}

Active contour model (ACM), or snake, is an efficient tool for image segmentation. It was originally introduced by Kass et al. [1]. Generally, there are two types of active contour model, i.e., parametric active contour model [1] and geometric active contour model [2]. The former represents curves and surfaces explicitly in their parametric forms. Compared with parametric active contour models, the geometric active contour model represents curves and surfaces implicitly as a level set of higher dimensional function [3]. This letter focuses on parametric active contour model.

A snake [1] is a parametric curve $v(s)=(x(s), y(s)), s \in$ $[0,1]$. The converged curve is viewed the object boundary by minimizing the following energy functional:

$$
E_{\text {snake }}=\int_{0}^{1} \frac{1}{2}\left(\alpha(s) v_{s}^{2}(s)+\beta(s) v_{s s}^{2}(s)\right)+E_{\text {ext }}(v(s)) d s
$$

where $v_{s}(s)$ and $v_{s s}(s)$ are the first and second derivatives of

Manuscript received April 3, 2013.

Manuscript revised July 13, 2013.

$\dagger$ The authors are with the School of Electronic and Information Engineering, South China University of Technology, Guangzhou, 510640, China.

*Presently, with the Graduate School of Information, Production and Systems, Waseda University, Kitakyushu-shi, 808-0135 Japan.

a) E-mail: liuguoqi080408@163.com

b)E-mail: zhouzh@scut.edu.cn

c) E-mail: eeoshlxie @ scut.edu.cn.

d) E-mail: dongcheng.wdc@gmail.com

DOI: $10.1587 /$ transinf.E96.D.2500 $v(s)$ with respect to arc length parameter $s$, the parameters $\alpha(s), \beta(s)$ are the weight factors and always set constants. The external energy $E_{\text {ext }}$ is derived from an image, which is usually computed as follows:

$$
E_{\text {ext }}(x, y)=-\left|\nabla\left[G_{\sigma}(x, y) * I(x, y)\right]\right|^{2}
$$

where $G_{\sigma}(x, y)$ is a two-dimensional Gaussian function with standard deviation $\sigma, I$ is the gray of the image. In calculus of variations [4], the Euler equation to minimize $E_{\text {snake }}$ is

$$
\alpha v_{s s}(s)-\beta v_{s s s s}(s)-\nabla E_{\text {ext }}=0
$$

The first two terms are always viewed as the internal force smoothing the contour while the last term $-\nabla E_{\text {ext }}$ is the external force pulling the contour to the desired features. The segmentation tasks will be turned into a process of curve evolution which is derived from an energy functional minimization. Affected by both internal force and external force, the parametric curve, i.e., the contour, moves to the direction of minimum energy.

The external force plays a leading role in the evolution of the contour and most of methods are concerned with the external force in parametric active contour models, such as balloons [5], vector field convolution (VFC) [6], gradient vector flow (GVF) [7], its generalization (GGVF) [8] and improvement [9]-[11]. VFC is calculated by convolving an vector field kernel with the edge map derived from an image. It has a large capture range and needs few computational cost. However, VFC has difficulty in extracting an object with deep concavity and complex geometries when contour is initialed outside the object or concave region.

In this letter, the correlation of the external forces generated by various edges in VFC is analyzed. Because of the correlation, VFC could not extract the concavity. In order to decrease the correlation of external force, a new type of external force for snakes, called dynamically constrained vector field convolution (DCVFC), is proposed. By introducing an indicator function with respect to the evolving contour, the edge information only inside the contour is used to convolute the vector field kernel to generate new external force. Thus, proposed force field is not affected by force generated by the edges outside evolving contour. The vector fields by dynamically convolving a vector field kernel with constrained edge map are obtained to attract the contour converge to concave region. 


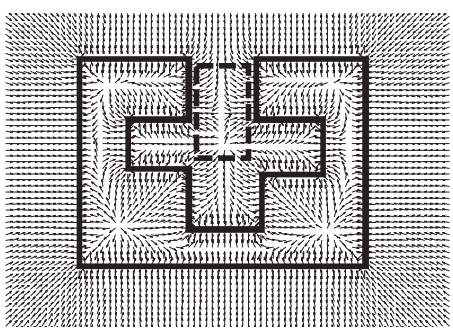

(a)

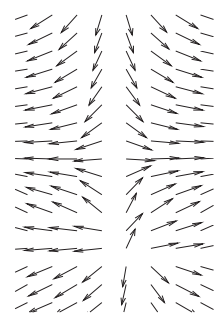

(b)
Fig. 1 Some conflict components appear in VFC. (a) VFC vector field; (b) The vector field component (zoomed) in the dotted rectangle of (a).

\section{Problem Analysis}

\subsection{Vector field convolution (VFC)}

Vector field convolution (VFC) is introduced by Li [6]. It was given by

$$
V^{v f c}(x, y)=k(x, y) \otimes f(x, y)
$$

where $f$ is the edge map derived from an image $I, f$ is given as follows:

$$
f=\left|\nabla\left[G_{\sigma}(x, y) \otimes I(x, y)\right]\right|^{2}
$$

$G_{\sigma}(x, y)$ is a two-dimensional Gaussian function with standard deviation $\sigma . \otimes$ is convolution operator, $k(x, y)$ is the vector field kernel defined by:

$$
k(x, y)=\left(\sqrt{x^{2}+y^{2}}+\epsilon\right)^{-\gamma} \cdot\left(\frac{-x}{\sqrt{x^{2}+y^{2}}}, \frac{-y}{\sqrt{x^{2}+y^{2}}}\right)
$$

where $\gamma$ is positive parameter to control the decrease, $\epsilon>0$ is a small positive constant to prevent division by zero at the origin. Let $r=\sqrt{x^{2}+y^{2}}, m=(r+\epsilon)^{-\gamma}$ and $\vec{n}(x, y)=$ $(-x / r,-y / r)$, then $k(x, y)=m(x, y) \cdot \vec{n}(x, y) . m(x, y)$ is the magnitude of the vector at $(x, y)$ and $\vec{n}(x, y)$ is the unit vector pointing to the kernel origin.

\subsection{Correlation Analysis of the External Forces Generated} by Various Edges

From the generation of VFC, the capture range of VFC is determined by the vector field kernel $k(x, y)$. However, with a large capture range in VFC, correlation of external forces derived from different edges results in saddle points [12]. Near the saddle points, some conflict components [13] in VFC stop the contour converging to concavity, which is shown in Fig. 1. It can be analyzed as follows: As shown the forces at a point $O$ in Fig. 2 (a), the forces generated by the various edges impose the point $O$. Force derived from the deepest concave edges which are far away the point $O$ is small and even could be ignored since the influence from the edge feature decreases as the particles are far away. Therefore, the external forces derived from concave boundary points (such as $E, F$ ) are overwhelmed by the forces generated by the other edges points (such as $A, B, C, D$ ). The
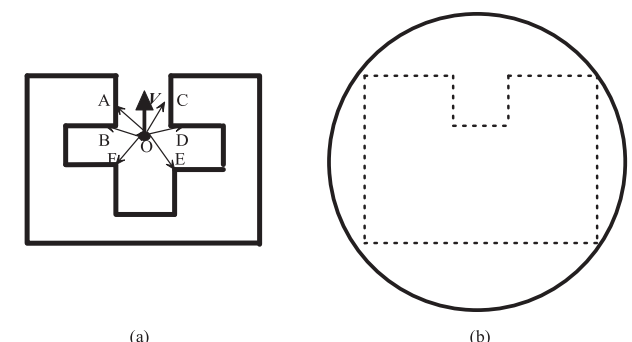

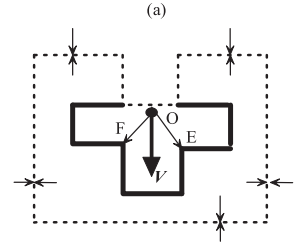

(c)

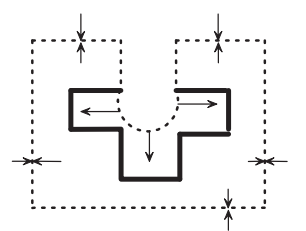

(d)
Fig. 2 (a) The motion of particle $O$ is determined by the resultant force $V$ generated by various edge points (such as $A, B, C, D, E, F$ ); (b) Initial contour (circle) deforms in VFC and stops undesired location (dashed line); (c) The resultant force $V$ at $O$ generated by the edge points (such as $E, F$ ) in DCVFC; (d) The contour moves to the object.

resultant force $V$ at $O$ does not point to the concave boundary because of correlation of the external forces generated by various edges.

\section{Proposed Method}

Based on the above analysis, DCVFC is proposed in this section. By introducing an indicator function, the vector field are generated by constrained edge gradient information. On the other hand, the proposed vector field is dynamic. But different from tradition dynamic fields [5], [12], DCVFC does not change at each iteration. It is composed by multistage vector fields. Waiting for the convergence in one stage of vector fields, then external field is updated. The converged contour is viewed as the initial contour in the next stage of vector fields.

\subsection{DCVFC External Forces}

Firstly, the first stage of vector fields $V_{1}$ is obtained by interpolating the vector field $V^{v f c}$ which is calculated by Eq. (4) and the traditional vector field $\nabla f$ as follows:

$$
V_{1}=\tau V^{v f c}+(1-\tau) \nabla f
$$

where $\tau=e^{-\frac{|\nabla f|}{K}}, K$ is a parameter. If the edges to preserve are weak, $K$ should be small and vice versa. Vector field $V^{v f c}$ dominates in the homogeneous region and the traditional one dominates near the object boundary. As shown in Fig. 2 (b), initial contour (the circle) is placed outside the object, converged contour (dashed line) could not completely extract the complex concavity in the $V_{1}$ stage.

Assuming the converged contour in $V_{1}$ is $C_{1}$, the indicator function $H_{C_{1}}$ is defined as follows:

$$
H_{C_{1}}(x, y)= \begin{cases}1, & (x, y) \text { is inside the } C_{1} \\ 0, & \text { otherwise }\end{cases}
$$


Then, the next stage external field $V_{2}$ is given as follows:

$$
V_{2}=\tau V_{2}^{v f c}+(1-\tau) \nabla f
$$

where $V_{2}^{v f c}$ is calculated by $V_{2}^{v f c}=k \otimes\left(H_{C_{1}} * f\right)$. Contour with the initial curve $C_{1}$ deforms in the vector field $V_{2}$ to extract the object. As shown in Fig. 2(c), because of the indicator function $H_{C_{1}}$, the edges outside or on the contour $C_{1}$ are not used to generate external forces. The resultant force $V$ at point $O$ generated by the edge inside the contour $C_{1}$ (such as $E, F$ ) point to the concave region. As shown in Fig. 2(d), the external force $V_{2}^{v f c}$ attracts the contour to the concavity, the vector field $\nabla f$ makes the contour stop at object boundary.

Generally, the proposed vector field $V_{i}$ can be uniformly represented as following:

$$
V_{i}=\tau V_{i}^{v f c}+(1-\tau) \nabla f, i=1,2,3, \cdots, n
$$

where $V_{i}^{v f c}=k \otimes\left(H_{C_{i-1}} * f\right), C_{i}$ is the converged contour in the vector field $V_{i}, n$ is an integer. When $i=1, H_{C_{0}}$ with respect to the initial contour $C_{0}$ is considered to 1 at every point. Thus, $V_{1}$ is the same as VFC in homogeneous region, it is robust to initialization. For complex geometries, the vector field is not generated until the contour is unchanged in the new vector field.

\subsection{Connections between VFC and DCVFC}

From the Eq. (7), VFC can be considered a special case of the vector field $V_{1}(\tau=1)$. On the other hand, VFC can be represented as:

$$
V^{v f c}=k \otimes f=k \otimes\left(H_{C} * f\right)+k \otimes\left(\left(1-H_{C}\right) * f\right)
$$

Thus, we have

$$
V_{v f c}=V_{i}^{v f c}+k \otimes\left(\left(1-H_{C_{i-1}}\right) * f\right)
$$

In homogeneous regions of the image, the second term in Eq. (10) can be ignored because the gradient of edge map $f$ is zero and $\tau=e^{-\frac{|\nabla f|}{K}}=1$. Therefore, $V^{v f c}$ is represented with $V_{i}$ in homogeneous region:

$$
V^{v f c}=V_{i}+k \otimes\left(\left(1-H_{C_{i-1}}\right) * f\right)
$$

From the above equation, $V^{v f c}$ could not make the contour move to concave region because of influence of the term $k \otimes\left(\left(1-H_{C_{i-1}}\right) * f\right)$. When initial contour is placed outside the object or concave region, the force $k \otimes\left(\left(1-H_{C_{i-1}}\right) * f\right)$ generated by the edges outside the contour $C_{i-1}$ make the contour away from the concavity. In proposed force field, external force generated by $k \otimes\left(\left(1-H_{C_{i-1}}\right) * f\right)$ is omitted. Therefore, the external forces generated by the edges inside the contour gradually attract the contour to the complex or concave region.

\section{Experimental Results}

In this section, the performance of the VFC snake and proposed snake are compared. The edge map $f$ used in two snakes is normalized to the range $[0,1], \alpha=0.2$ and $\beta=0.1$ are set in both snakes for all experiments. For each tested image, the same initialization is employed for the two snakes. The parameter $K$ for proposed snake is set to 0.01 in all experiments unless otherwise stated. Because VFC can be viewed a special case of the DCVFC, DCVFC has the desirable properties of the VFC vector field, such as initialization insensitiveness. It also improves the performances of VFC snake in extracting the deep concavity and complex geometries, which are verified in this section.

\subsection{Deep and Complex Concavity Extracting}

A 20-pixel long, 5-pixel wide concavity is employed to test the performance of the VFC and DCVFC snakes. The first row of Fig. 3 (c) and (d) shows that the proposed snake succeeds in extracting the deep and narrow concavity by evolving two stage of vector fields. Contour evolves 125 times in VFC and every stage of DCVFC. In VFC, the forces generated by the concavity are affected by the forces generated by other edges, the VFC snake could not generate enough forces to push the contour converge to the deepest concavity, which is shown in the first row of Fig. 3 (b).

Two images with complex concave geometries are also used to test the performance of VFC and DCVFC snakes. In VFC, there are "equilibrium issues" [12] in dealing with the complex geometries. As shown in the second row of Fig. 3 (a), initial contour has crossed the object. In VFC, the forces generated by edges have the conflict components. Thus, contour suffers from local minimum with VFC snake, which is shown in second row of Fig. 3 (b). From the connections between VFC and DCVFC force fields, DCVFC is also robust to initialization. As shown the second row of Fig. 3 (c) and (d), DCVFC snake makes the contour completely converge to the object.

In the last row of Fig. 3, the object with a cross-
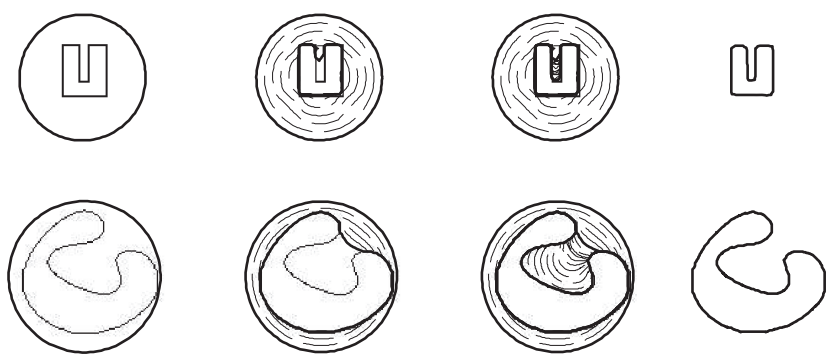

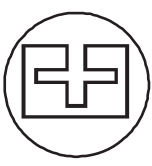

(a)

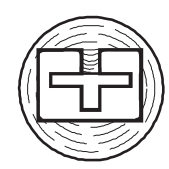

(b)

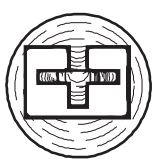

(c)

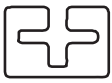

(d)
Fig. 3 Performance of VFC and DCVFC snake on image with deep concavity and complex geometries. (a) Original image with the initial snake position indicated (circles); (b) convergence of the VFC snakes; (c) convergence of the DCVFC snakes; (d) the final results with DCVFC snakes. 


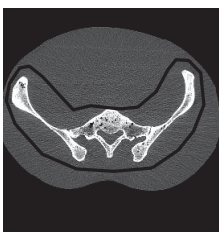

(a)

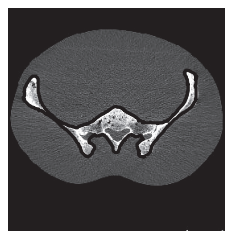

(b)

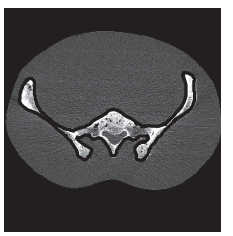

(c)
Fig. 4 Performance of VFC and DCVFC snake on gray image. (a) Original image with the initial snake position indicated. Result (b) with VFC snake and (c) with DCVFC snake.

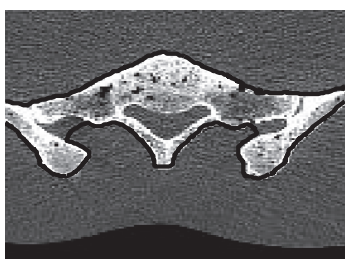

(a)

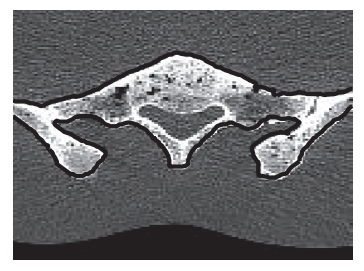

(b)
Fig.5 Magnified results at concavity with VFC and DCVFC snakes. (a) Result with VFC snake and (b) result with DCVFC snake.

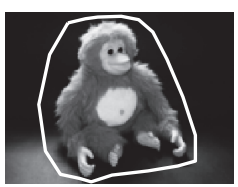

(a)

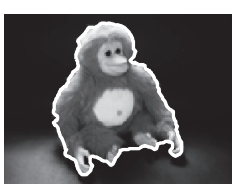

(b)

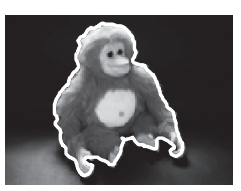

(c)
Fig. 6 Performance of VFC and DCVFC snake on gray image. (a) Original image with the initial snake position indicated. (b) Result with VFC snake and (c) result with DCVFC snake.

shaped concave boundary is also tested. VFC suffers from premature convergence, the contour stops undesired location. While the contour with DCVFC snake converges to the cross-shaped concave boundary by deforming in three stages of vector field $\left(V_{1}, V_{2}\right.$ and $\left.V_{3}\right)$. The final result with proposed method is shown in the final row of Fig. 3 (d).

\subsection{Gray Images}

Figure 4 shows the segmentation results of the VFC and DCVFC snakes on gray image. The $512 * 512$ medical image is polluted by noise and there are some deep concavities. The contour initialed near the object boundary. Compared with VFC snake, DCVFC snake successfully converges to the concavities, which is shown in Fig. 4 (c) and Fig. 5 (b). Another $160 * 120$ gray image is also tested. As shown in the Fig. 6, DCVFC snake also converges to the boundary of the monkey. While VFC snake failed to extract the concavity, especially the hand of the monkey.

\section{Conclusion}

In this letter, the DCVFC external force is proposed. The constrained edges gradient information is used to remove the correlation of the external forces generated by various edges. On the other hand, the proposed external field is dynamic. Experimental results on synthetic and gray images show that DCVFC snake outperforms VFC snake. This algorithm is particularly useful for extracting the object with deep and complex concavity.

\section{Acknowledgments}

This work was supported by National Natural Science Foundation of China (grant 61003170, 61372142), China Postdoctoral Science Foundation (grant 2012M511561), Fundamental Research Funds for the Central Universities SCUT (grant 2012ZZ0033), Guangdong Province Science Foundation (grant S2011010001936).

\section{References}

[1] M. Kass, A. Witkin, and D. Terzopoulus, "Snakes: Active contour model," Int. J. Comput. Vis., vol.1, no.4, pp.321-331, 1988.

[2] V. Caselles, F. Catte, T. Coll, and F. Dibos, "A geometric model for active contours," Numer. Math, vol.66, pp.1-31, 1993.

[3] R. Kimmel, A. Amir, and A. Bruckstein, "Finding shortest paths on surfaces using level set propagation," IEEE Trans. Pattern Anal. Mach. Intell., vol.17, no.6, pp.635-640, June 1995.

[4] G. Aubert and P. Kornprobst, Mathematical problems in image processing: Partial Differential Equations and the Calculus of Variations, Springer, New York, 2006.

[5] L. Cohen and I. Cohen, "Finite-element methods for active contour models and balloons for 2D and 3D images," IEEE Trans. Pattern Anal. Mach. Intell., vol.15, no.11, pp.1131-1147, Nov. 1993.

[6] B. Li and S.T. Acton, "Active contour external force using vector field convolution for image segmentation," IEEE Trans. Image Process., vol.16, no.8, pp.2096-2106, Aug. 2007.

[7] C. Xu and J. Prince, "Snakes, shapes, and gradient vector flow," IEEE Trans. Image Process., vol.7, vol.3, pp.359-369, March 1998.

[8] C. Xu and J. Prince, "Generalized gradient vector flow external Forces for active contours," Signal Process., vol.71, vol.2, pp.131139, 1998.

[9] J. Cheng and S.W. Foo, "Dynamic directional gradient vector flow for snakes," IEEE Trans. Image Process., vol.15, no.6, pp.1653$1671,2006$.

[10] J. Ning, C. Wu, S. Liu, and S. Yang, "NGVF: An improved external force field for active contour model," Pattern Recognit. Lett., vol.28, pp.58-63, 2007.

[11] Y. Wang, L. Liu, H. Zhang, Z. Cao, and S. Lu, "Image segmentation using active contouts with normally basied GVF external force," IEEE Signal Process. lett., vol.17, no.10, pp.875-879, 2010.

[12] X. Xie and M. Mirmehdi, "MAC: Magnetostatic Active contour model," IEEE Trans. Pattern Anal. Mach. Intell., vol.30, no.4, pp.632-647, April 2008.

[13] S.L. Xie, D.L. Zeng, Z.H. Zhou, and J. Zhang, "Arranging and interpolating sparse unorganized feature points with geodesic circular arc," IEEE Trans. Image Process., vol.18, no.3, pp.582-595, March 2009. 